\title{
Endoscopic ultrasound-guided gallbladder drainage: a technical review
}

\section{Surinder S. Rana}

Postgraduate Institute of Medical Education and Research (PGIMER), Chandigarh, India

\section{Abstract}

\begin{abstract}
Endoscopic ultrasound-guided gallbladder drainage (EUS-GBD) has emerged as a safe and effective minimally invasive alternative to both percutaneous and endoscopic transpapillary GBD in patients with acute cholecystitis. Over the last few years, the technique, as well as the indications for EUS-GBD have been gradually evolving, and the procedure has become simpler and safer as the accepted indications have expanded. The development of lumen-apposing metal stents (LAMS) has allowed us to realize the dream of creating endoscopic gastrointestinal anastomoses, and has thus paved the way for a safer EUS-GBD. Single step EUS-guided LAMS delivery systems have obviated the use of other endoscopic accessories and thus made EUS-GBD simpler and safer. However, EUS-GBD can be associated with potentially serious complications, and therefore should be performed by expert interventional endosonologists at centers with surgical and radiological back up. EUS-GBD is a relatively new procedure still in its infancy, but continued improvement in EUS accessories and dedicated stents will make this procedure safer and also expand its current indications. This review focuses on the technical aspects, including procedural details, as well as the complications of EUS-GBD.
\end{abstract}

Keywords Stent, endosonography, magnetic resonance cholangiopancreatography, endoscopic retrograde cholangiopancreatography, lumen-apposing metal stent

Ann Gastroenterol 2021; 34 (1): 1-7

\section{Introduction}

Over the last 3 decades endoscopic ultrasound (EUS) has come a long way from a pure diagnostic procedure to a safe and effective interventional procedure. Successful EUS-guided drainage of pancreatic fluid collections has been a watershed moment in the field of interventional EUS, igniting a wide plethora of EUSguided transmural interventions, including biliary, pancreatic duct and gallbladder drainage (GBD) [1-3]. Although exciting, these transmural interventions were initially associated with high complication rates because of a lack of dedicated accessories and stents. The development of lumen-apposing metal stents (LAMS) and single-step EUS-guided delivery systems has made these procedures safer and faster, and has inaugurated the new field of EUS-guided anastomotic interventions [4].

Department of Gastroenterology, Postgraduate Institute of Medical Education and Research (PGIMER), Chandigarh, India

\section{Conflict of Interest: None}

Correspondence to: Prof. Surinder S. Rana, Department of Gastroenterology, Postgraduate Institute of Medical Education and Research (PGIMER), Chandigarh 160012, India,

e-mail: drsurinderrana@yahoo.co.in

Received 13 July 2020; accepted 5 October 2020; published online 4 January 2021

DOI: https://doi.org/10.20524/aog.2020.0568
EUS-GBD has emerged as a safe and effective minimally invasive alternative to both percutaneous and endoscopic transpapillary GBD [2]. Percutaneous drainage had been the conventional non-surgical treatment modality for GBD in patients with acute cholecystitis. Although effective, it has been associated with various limitations, including complications such as bleeding, bile leak, biliary peritonitis, pneumothorax, and bowel perforation, as well as secondary infections, patient discomfort, and risk of accidental dislodgement $[5,6]$. Moreover, in certain clinical situations, such as patients on anticoagulation or with significant ascites, percutaneous GBD is not technically possible. Endoscopic transpapillary drainage is a safe and effective alternative to percutaneous drainage, with the added advantage that the GBD is internal, thus overcoming the problems associated with external drainage [7-9]. However, it is a technically challenging procedure and fails in a significant number of patients, because selective cannulation of the cystic duct is prevented by cystic duct obstruction due to stone or inflammation. Transpapillary drainage is also associated with an increased risk of post-endoscopic retrograde cholangiopancreatography (ERCP) pancreatitis.

EUS-GBD is a newly developed, minimally invasive procedure for internal GBD. A systematic review, together with randomized controlled studies comparing EUS-GBD with percutaneous GBD, have demonstrated similar technical and clinical success for both procedures. However, EUS-GBD is associated with significantly fewer adverse effects, lower post-procedure pain scores, lower analgesic requirements, a 
shorter hospital stay, and fewer unplanned readmissions and reinterventions [10-12]. EUS-GBD has also been demonstrated to be superior to endoscopic transpapillary GBD. A systematic review and meta-analysis comparing the 2 procedures reported that EUS-GBD had significantly higher technical as well as clinical success rates, associated with similar adverse effects and lower rates of recurrent acute cholecystitis [13]. Therefore, in centers where the expertise is available, EUSGBD is considered as the procedure of choice for GBD in highrisk surgical patients with acute cholecystitis [14]. This review focuses only on the technical aspects, including procedural details, as well as the complications of EUS-GBD, and will not discuss the clinical outcomes of EUS-GBD or how its results compare with those of percutaneous and endoscopic transpapillary drainage.

\section{Applied anatomy of the gallbladder}

The gallbladder is a pear-shaped hollow organ located anteriorly on the under surface of segments IV and $\mathrm{V}$ of the liver $[15,16]$. The gallbladder is divided into fundus, body and neck, with the neck opening into the cystic duct. The fundus extends beyond the inferior border of the liver, behind the ninth costal cartilage. The body of the gallbladder continues upwards from the fundus and is directed posteriorly and to the left. The body is continuous, with the neck at the right border of the porta, coursing posteriorly and inferiorly into the cystic duct. EUS-GBD is performed either from the stomach or the duodenum. The body of the gallbladder lies anterolateral to the distal first part and proximal second part of the duodenum, and the neck of the gallbladder lies anterosuperior to the first part of the duodenum. The neck of the gallbladder is the preferred site for transduodenal GBD, as it is less mobile and located close to the duodenal wall. In contrast, the body of the gallbladder is the preferred site for transgastric puncture, because of its close proximity to the antrum of a distended stomach [17-19].

\section{Indications for EUS-GBD}

EUS-GBD is a relatively new procedure and its accepted indications are gradually expanding [5,6,18-21]. The current indications for EUS-GBD in patients with acute cholecystitis are:

1. Patients unfit for surgery. This can be combined with trans-stent stone extraction (peroral cholecystoscopic interventions).

2. Bridge to surgery and alternative to percutaneous drainage in patients unsuitable for emergency cholecystectomy. EUS-GBD should be performed in centers where expertise in therapeutic EUS as well as subsequent cholecystectomy with stent in situ is available.

3. As an alternative to either failed percutaneous or endoscopic transpapillary GBD.

4. As an alternative to EUS-guided biliary drainage in patients with malignant distal biliary obstruction.
5. Significant perihepatic ascites rendering percutaneous drainage difficult.

6. Conversion of external percutaneous drainage to internal endoluminal drainage.

7. Patients with coagulopathy or on antithrombotic/ antiplatelet medication [22,23]. Case series have reported that EUS-GBD is safe, feasible, and is not associated with an apparent increased risk of bleeding in patients with prolonged prothrombin time and/or on anticoagulation.

Although there are no described contraindications for the procedures, the presence of significant ascites or severe coagulopathy maybe considered as a relative contraindication [21]. Inability to visualize a gallbladder in close apposition to the stomach or duodenum would preclude EUS-GBD.

\section{Echoendoscope and accessories}

As with all complex EUS-guided interventional procedures, EUS-GBD is usually done under monitored anesthesia, with or without endotracheal intubation. We have been performing EUS-GBD under conscious sedation with adequate analgesia. All patients should receive both pre- and postprocedural antibiotics. A conventional curved linear array oblique-viewing therapeutic echoendoscope is usually used for performing EUSGBD. A forward viewing echoendoscope can also be used for EUS-GBD, with the added advantage of inserting force being applied directly with the scope towards the target, rather than the tangential dilating force applied using the linear echoendoscope [24]. However, the absence of an elevator is a significant limitation of this scope, and we recommend using a curved linear array oblique-viewing therapeutic echoendoscope for EUS-GBD. The procedure is performed under combined EUS and fluoroscopic guidance. The accessories required are a 19-G EUS fine-needle aspiration (FNA) needle, a 0.025/0.035-inch guidewire, a tract-dilating accessory (electrocautery dilating device: cystotome, needle knife or electrocautery-enhanced stent delivery system, or a non-cautery dilating device such as tapered cannulas, stepped axial dilators or dilating balloon) and appropriate stents [2,6,17,20,21,25-27].

Studies have reported using various endoprostheses for EUSGBD, such as plastic pigtail stents, nasobiliary drain, fully covered metallic stents (FCSEMS) and LAMS. The pooled technical as well as clinical success rates for plastic stents, FCSEMS and LAMS have been reported to be comparable (pooled technical success rates for plastic stents, self-expanding metallic stent (SEMS), and LAMS: 100\%, 98.6\%, and $91.5 \%$, respectively; pooled clinical success rates for plastic stents, SEMS, and LAMS: $100 \%, 95.5 \%$, and $90.1 \%$, respectively) (Table 1) [28]. However, the $100 \%$ success rates with plastic stents are an overestimation, as these data come mainly from case reports with a short-term follow up. Furthermore, plastic stents are associated with a high risk of bile leaks. The potential advantages of SEMS over plastic stents are their larger diameter and the immediate sealing of the transmural fistulous tract after stent expansion, thus avoiding bile leak. However, FCSEMS are long, non-apposing and associated with a high risk of stent migration. Therefore, neither plastic 
Table 1 Comparison of various types of stents for endoscopic ultrasound-guided gallbladder drainage as reported in a systematic review by Anderloni et al [20]

\begin{tabular}{lccc}
\hline Parameters & Plastic stent & SEMS & LAMS \\
\hline Number of patients & $1-8$ & $1-63$ & $1-30$ \\
Technical success & $100 \%$ & $98-100 \%$ & $84-100 \%$ \\
Clinical success & $100 \%$ & $66-100 \%$ & $84-100 \%$ \\
Adverse effects & $13.6-33 \%$ & $4.8-33 \%$ & $8-15 \%$ \\
Pneumoperitoneum & 1 patient & 2 patients & - \\
Bile peritonitis & 1 patient & - & - \\
Bile leakage & 1 patient & - & - \\
Stent migration & 1 patient & 3 patients & - \\
Duodenal perforation & - & 1 patient & - \\
Bleeding & - & - & 3 \\
\hline${ }^{*}$ Case reports and series only & & & \\
SEMS, self-expanding metal stent; LAMS, lumen-apposing metal stent
\end{tabular}

stents nor FCSEMS are usually preferred for transluminal GBD. To overcome the limitation of stent migration associated with FCSEMS, partially covered SEMS (PCSEMS), with uncovered flared ends for preventing stent migration by tissue ingrowth, have also been used. However, the uncovered ends of PCSEMS can potentially cause mucosal injury, bleeding and stent embedding [28,29]. LAMS (AXIOS - Boston Scientific, Mattick, MA, USA; NAGI - Taewoong, Gimpo, Korea; and SPAXUS - Taewoong, Gimpo, Korea) permit the creation of a closely apposing anastomosis and are therefore the stents of choice for EUS-GBD. LAMS are available in diameters of $6,8,10,12,14$, 15,16 or $20 \mathrm{~mm}$, with the usual length being $10 \mathrm{~mm}$ and smaller caliber LAMS (10 $\mathrm{mm}$ or $8 \mathrm{~mm}$ if available) being preferred for GBD. However, larger LAMS (15 mm) may be preferred if peroral cholecystoscopic interventions are planned, where the endoscope is to be inserted into the gallbladder through the LAMS [30]. The NAGI stent has a lower apposing force compared to other LAMS, and most of the studies on EUS-GBD have used the AXIOS stent. However, Manta et al have reported that the use of a NAGI stent for EUS-GBD is safe and effective, with technical and clinical success rates comparable to the pooled success rates for AXIOS [31]. The development of single-step EUS-guided LAMS delivery systems (Hot AXIOS, Hot SPAXUS and Hot NAGI stents) has made EUS-guided drainage procedures simpler, and using a freehand insertion technique the procedure can be completed quickly without the use of any other accessory. However, EUS-GBD is a complex procedure, as the gallbladder is a mobile structure and there is therefore a risk of it moving away from the stomach or duodenum during free-hand insertion of LAMS, leading to perforation. Therefore, the one-step, free-hand technique should be used only by experts, while beginners should use the over-thewire technique for stent insertion. A hybrid free-hand LAMS insertion technique has also been described that combines the speed of the free-hand technique with the safety of the over-thewire technique. In this technique, the guidewire is preloaded into the cautery-enabled stent delivery catheter and is coiled into the gallbladder after free-hand entry [32]. The stent is deployed only after the guidewire has been securely coiled into the gallbladder. The proponents of the free-hand technique argue that placement of the guidewire can potentially push the mobile gallbladder wall away from the gastric or duodenal wall, and that this can increase the risk of maldeployment of the LAMS, as well as bile leakage.

\section{Pre-drainage evaluation}

The cross-sectional imaging, including computed tomography or magnetic resonance imaging, should be carefully evaluated for detailed gallbladder anatomy, its relations with the stomach and duodenum, as well as anatomical variations of the gallbladder. A preprocedural interdisciplinary consultation involving the endoscopist, interventional radiologist and surgeon should discuss a detailed management plan, as well as a rescue plan in case of failure or adverse events. Moreover, before embarking upon drainage, the gallbladder should be carefully evaluated on EUS from both antrum and duodenum, identifying various parts of the gallbladder as well as the degree of distension. The gallbladder should be carefully evaluated for any abnormal wall thickening or mass. The gastric or duodenal mucosa should also be carefully evaluated for any ulceration or malignant infiltration. The infiltrated mucosa is hypervascular, which increases the risk of periprocedural bleeding [26]. The site of intended puncture should be evaluated with Doppler to define the surrounding vascular anatomy and detect any abnormal vessels.

\section{Site of puncture: stomach or duodenum?}

As mentioned above, the gallbladder neck is usually accessed from the duodenum and gallbladder body, or occasionally the fundus is accessed through the stomach. Theoretically, both transduodenal and transgastric GBD have potential advantages. The duodenum is a less mobile structure, as well as being closer to the gallbladder compared to the stomach; therefore, there is theoretically a lower risk of stent migration. Moreover, transduodenal GBD is associated with a smaller risk of food reflux. In contrast, transgastric puncture of the gallbladder is easy, because of the larger diameter of the gallbladder body. Moreover, the larger diameter of the gallbladder body allows easier deployment of the inner flange of the LAMS. Moreover, in case of a failed procedure, rescue surgery is much easier in patients with transgastric attempted drainage compared to transduodenal drainage [5,6,17-20,24-27,32-40]. Limited studies comparing transgastric with transduodenal EUS-GBD have reported no significant differences in technical or clinical success rates or in the frequency of complications [30,41]. Patients in whom EUS-GBD has been performed as a bridge to cholecystectomy have an important issue of technical difficulty in subsequent elective cholecystectomy. Some surgeons consider closing the transduodenal tract to be more difficult in comparison to the transgastric tract, whereas other surgeons consider the presence of a fibrous band between stomach and gallbladder following transgastric drainage a significant hindrance in subsequent elective cholecystectomy [32]. Hence, the available evidence 
suggests that both transgastric and transduodenal GBD are equally safe and effective; therefore, the route of drainage should be individualized depending upon anatomical and technical considerations. Transgastric drainage is preferred in patients where there is involvement of duodenum with malignancy, or with an indwelling duodenal SEMS. In the absence of these factors, the site of GBD should be individualized depending upon the site of closest apposition of the gallbladder to the gastrointestinal lumen, the absence of blood vessels around the puncture site, and stable positioning of the echoendoscope.

\section{Procedural details}

The first step in EUS-GBD is stabilizing the position of the echoendoscope. This is usually accomplished in a long scope position in most patients [18]. After the scope has been stabilized, the gallbladder is punctured using a 19-G EUS FNA needle (Fig. 1). The stylet is then removed and $2-3 \mathrm{~mL}$ of bile is aspirated to confirm the position of the needle tip. This is followed by injection of 2-3 mL of contrast medium to delineate the gallbladder. Next, a 0.025 - or 0.035 -inch guidewire is passed through the needle and coiled into the gallbladder, followed by dilatation of the transmural tract using either a bougie (6 Fr or $7 \mathrm{Fr}$ ) or tapered tip balloon dilator $(4 \mathrm{~mm})$. If there is resistance to dilation using non-cautery methods, a needle knife or a 6-Fr cystotome can be used. If a cauterybased dilating accessory is used, a coaxial dilating system like the cystotome should be preferred, because of the lower risk of perforation. Following needle puncture and dilatation, the stent is placed under EUS and fluoroscopic guidance. The step where the tract dilating device is inserted, followed by the stent
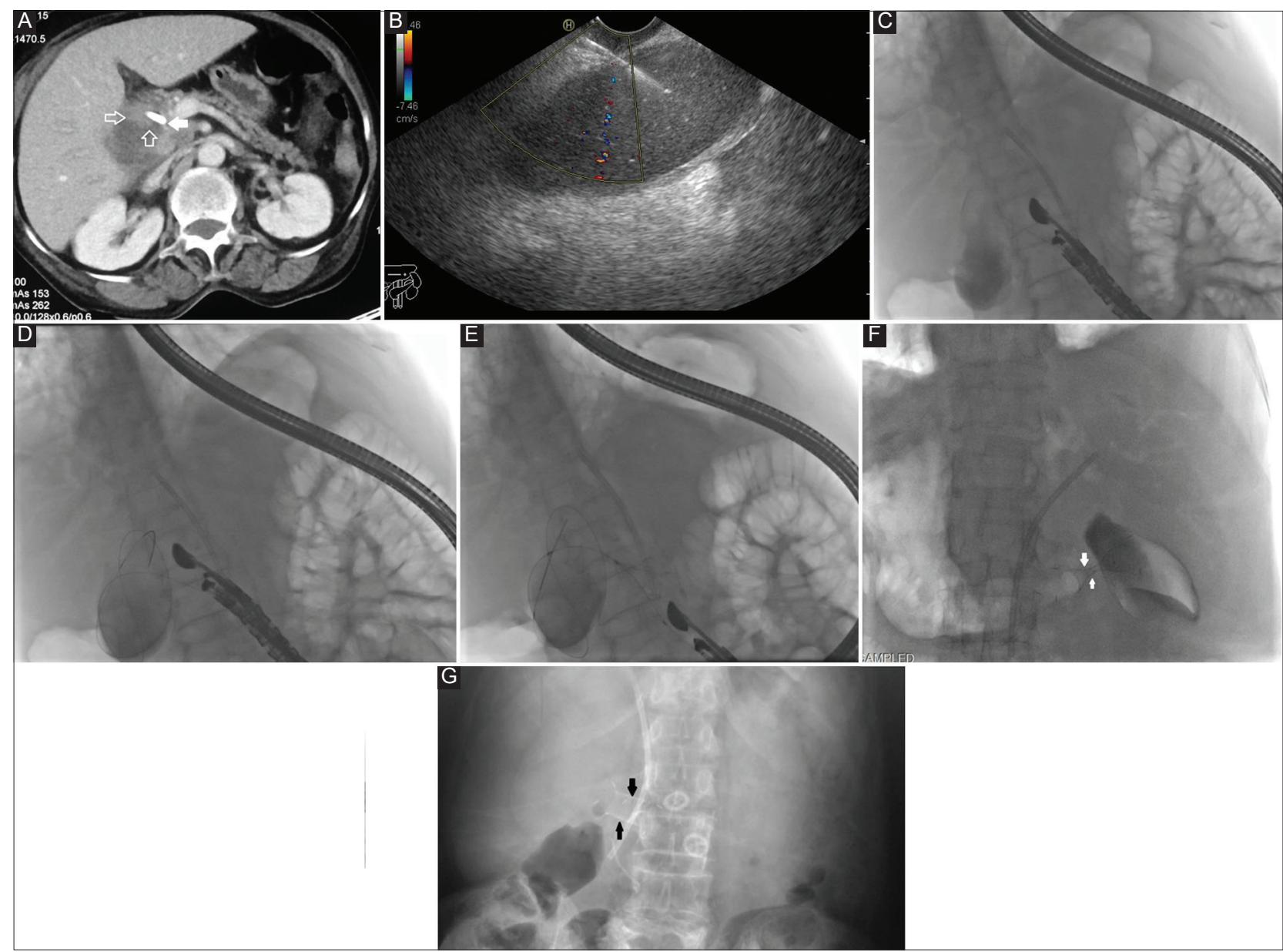

Figure 1 Stepwise EUS-guided drainage of the gallbladder. (A) Detailed evaluation of pre-procedure CT: gallbladder carcinoma located at neck with extra-hepatic biliary obstruction. A 10-Fr plastic biliary stent was placed. The jaundice was relieved but the patient presented with acute cholecystitis. CT shows infiltrated neck area of gallbladder (open arrows); therefore, the gallbladder body was chosen as the site of puncture. Plastic stent in CBD is also noted (bold arrow). (B) EUS-guided puncture of the gallbladder body through the antrum of the stomach. No blood vessels seen on color Doppler in the needle tract. (C) EUS cholecystogram obtained after injection of contrast into the gallbladder using a 19-G needle. Note stable long scope position. (D) The guidewire coiled in the gallbladder, maintaining stable scope position. (E) The transmural tract dilated using a 4-mm biliary balloon. The scope tends to fall back because of the resistance encountered while pushing the balloon into the gallbladder. (F) LAMS (Nagi stent; 12-mm diameter) deployed (arrows) connecting the gallbladder body with the gastric antrum. Note the waist of the stent (arrows) at the site of anastomosis. The tract was not dilated. (G) Abdominal X-ray $72 \mathrm{~h}$ after procedure shows fully expanded transmural stent EUS, endoscopic ultrasound; CBD, common bile duct; LAMS, lumen-apposing metal stent; CT, computed tomography 
delivery system, is a critical step in EUS-GBD. Sometimes, the dilating device may not penetrate the gallbladder wall but slide along its surface, despite the guidewire being coiled within its lumen. This usually happens when the direction of the dilating device is tangential to the gallbladder wall. This change in direction of the guidewire is usually due to its excessive coiling in the gallbladder [40].

LAMS can also be deployed using a single-step EUSguided LAMS delivery system. Using a free-hand technique, the gallbladder is punctured using a cautery-enhanced device, and the LAMS delivery catheter is subsequently passed inside the gallbladder lumen. The proximal flange of the stent is then deployed under EUS guidance, followed by slight retraction of the delivery system to create an apposition, and then deployment of the distal flange under endoscopic guidance, with or without fluoroscopic guidance. After the LAMS has been deployed, a double pigtail plastic stent may be placed within the LAMS for stent stabilization and prevention of tissue overgrowth [25]. We do not routinely place a plastic stent inside the LAMS; a plastic stent is placed only if the LAMS is intended to remain in situ for a prolonged period. The inner flange of the LAMS can theoretically cause mucosal bleeding by repeated abrasions of gallbladder mucosa, and a plastic stent deployed inside the LAMS can prevent this abrasion [26]. A 7-Fr, 3- or 4-cm double pigtail plastic stent can be placed if a $10-\mathrm{mm}$ LAMS/FCSEMS is used, and a 10-Fr stent can be used for larger diameter LAMS/FCSEMS. The LAMS may also be dilated after deployment to facilitate rapid GBD, but there is a lack of studies evaluating the efficacy and safety of stent dilatation post-deployment.

In patients with surgically altered anatomy, EUS-GBD may be performed between stomach and gallbladder (cholecystogastrostomy) or jejunum and gallbladder (cholecystojejunostomy) [26]. The choice of lumen for internal GBD is based on operator preference, patient-specific anatomy, and the proximity of gallbladder to the lumen; therefore, careful evaluation of cross-sectional imaging is of paramount importance for deciding on and appropriate puncture site. EUS-GBD is also an option for drainage in patients who have a malignant distal biliary obstruction with a patent cystic duct and surgically altered anatomy, where ERCP is not possible and EUS-guided biliary drainage is either not feasible or has failed [42].

\section{Complications of EUS-GBD}

EUS-GBD is a relatively safe procedure, with minimal complications in expert hands. However, studies have reported a significant number of procedural complications, with pooled analyses reporting complication rates varying between $8-17 \% \quad[5,17,18,20,30,33,41]$. These include bile leakage, stent migration into gallbladder or peritoneum, bleeding, gastroduodenal perforation, pneumoperitoneum, and recurrent acute cholecystitis due to stent occlusion [18]. Pneumoperitoneum is one of the most common complications and it has been speculated that it may be due to sheer dilation of the gallbladder [19]. Therefore, minimal dilating force is to be applied during dilatation of the transmural tract. The risk of pneumoperitoneum can also be reduced by using carbon dioxide for endoscopic insufflation.

Stent displacement or maldeployment is the most dreaded complication of EUS-GBD. The stent may either get completely deployed in the stomach or duodenum, or may migrate outward into the peritoneum. The outward migration of the stent into the peritoneum usually occurs in situations where the stent is being deployed in the gallbladder neck and the echoendoscope is in an unstable position. Because of limited space in the gallbladder neck area, the distal flange of the stent is clamped and relatively fixed. Coupled with an unstable echoendoscope position, any attempt to release the stent by pulling the sheath of the delivery system leads to paradoxical forward movement of the echoendoscope. This optical illusion makes the endoscopist think that the delivery sheath has been pulled back sufficiently for intra-scope channel release of the stent. This leads to maldeployment of the stent into the peritoneal cavity [40]. The most important trick for preventing and managing stent maldeployment is to ensure that the guidewire remains in place until proper stent deployment has been confirmed. In cases of stent maldeployment, the presence of the guidewire in place allows endoscopic rescue maneuvers to be performed safely and effectively [26]. The rescue maneuver when one end of the LAMS has been properly deployed on either the luminal or gallbladder side and the other end is maldeployed outside, is placement of an additional LAMS or longer FCSEMS through the previously maldeployed stent. The loss of the guidewire along with a maldeployed stent is a tricky situation, and usually requires percutaneous GBD, with or without peritoneal drainage, along with closure of the luminal perforation with clips or surgical rescue. The gastric perforation is easier to manage with gastric decompression using Ryle's tube and keeping the patient on nil orally for a few days. However, duodenal perforation usually requires surgical closure or endoscopic closure. Maldeployment of the stent into the peritoneum may require salvage surgery.

\section{What is the optimal duration of transmural stenting?}

In patients unfit for surgery and have limited life expectancy, LAMS can be left in situ and can provide adequate drainage for months, thereby preventing recurrence of acute cholecystitis [6,32]. Patients with no comorbidities should undergo elective cholecystectomy. Preliminary data suggest that the presence of a LAMS does not cause significant interference in cholecystectomy [43]. A comparative study that evaluated the results of elective cholecystectomy after EUSGBD or percutaneous GBD reported no difference between the 2 groups in the rate of conversion from laparoscopic to open cholecystectomy or in complications [43]. In patients unfit for surgery but have normal life expectancy, LAMS can be left for an indefinite period or replaced with a double pigtail plastic stent 4-6 weeks later, when the fistula tract has matured. The presence of a LAMS in situ for an indefinite 
period may have multiple harmful consequences, including the risk of recurrent cholecystitis due to stent blockage with food residue, gallbladder mucosal abrasion by stent flanges with a consequent risk of bleed, and the consequences of gastric reflux into the gallbladder $[32,44]$. Replacing the metal stent with a plastic stent 4-6 weeks later has been shown to be a reasonable strategy for reducing the risk of recurrent cholecystitis, as well as obviating the potential complications mentioned above [45].

An alternative to permanent transmural stenting would be to treat the underlying gallbladder disease and thereby obviate the need for long-term GBD. Use of a large-diameter (15 mm) LAMS for EUS-GBD allows repeated endoscopic access to the gallbladder for per-oral cholecystoscopy, stone removal, lithotripsy and other advanced interventions [46]. Moreover, after complete clearance of the gallbladder, the LAMS can be removed, thereby obviating the need for long-term stenting. The long-term consequences of this approach need to be studied prospectively, as there is a risk of recurrence of gallbladder stones. A previous study with a 10-year follow up of 439 patients successfully treated with percutaneous cholecystolithotomy reported recurrence of gallbladder stones in $41.2 \%$ of patients [47]. However, only $2 \%$ of patients were symptomatic and $7.5 \%$ of patients required cholecystectomy. Therefore, the results of this study suggest that cholecystectomy can be avoided in the majority of patients, despite the recurrence of stones. The risk of malignancy in the gallbladder is another issue that needs to be prospectively studied in patients who have been successfully treated with EUS-GBD and/or per oral cholecystoscopy.

\section{Concluding remarks}

EUS-GBD has emerged as an accepted minimally invasive non-surgical therapeutic option in centers with expertise for the management of high-risk patients with acute cholecystitis. The procedure involves visualization of the gallbladder on EUS, followed by puncture with a 19-G needle, coiling of a guidewire within the gallbladder, transmural tract dilatation and deployment of a stent (Table 2). The development of single-step EUS-guided LAMS delivery systems has made the procedure simpler, and using a free-hand insertion technique the procedure can be completed quickly as well as safely without using any other accessory. However, EUS-GBD can be associated with potentially serious complications, and therefore should be performed by expert interventional endosonologists at centers with surgical and radiological backup. EUS-GBD is a relatively new procedure still in its infancy, and continued improvement in EUS accessories and dedicated stents will make this procedure safer, while expanding its indications. There are many unanswered questions on various aspects of EUS-GBD, including the best type of stent, the preferred site of gallbladder puncture, and the long-term consequences and management of both stent and gallbladder after resolution of symptoms. Larger, prospective, multicenter studies are required to answer these questions. Based upon current evidence, it appears that EUS-guided GBD is slowly inching towards prime time!
Table 2 Summary of key concepts of endoscopic ultrasound-guided gallbladder drainage (EUS-GBD)

- EUS-GBD is a safe and effective minimally invasive alternative to percutaneous or endoscopic transpapillary gallbladder drainage

- EUS-GBD can be performed via either a transgastric or a transduodenal route. The neck of the gallbladder is the preferred site for transduodenal drainage and the body of the gallbladder is the preferred site for transgastric puncture

- EUS-GBD seems to be safe, with no apparent increase in the risk of bleeding in patients with abnormal coagulation parameters

- Lumen-apposing metal stents (LAMS) permit the creation of closely apposing anastomoses and are therefore the stents of choice for EUS-GBD

- A preprocedural interdisciplinary consultation, involving the endoscopist, interventional radiologist and surgeon, is critical for performing a successful EUS-GBD

- The site of gallbladder drainage should be individualized depending upon the site of closest apposition to the gastrointestinal lumen, absence of blood vessels in the transmural tract and stable positioning of the echoendoscope

- The insertion of the tract dilating device followed by the stent delivery system is a critical step in EUS-GBD

- The development of single-step EUS-guided LAMS delivery systems has made the procedure simpler

- Bile leakage, stent migration into the gallbladder or peritoneum, bleeding, gastroduodenal perforation, pneumoperitoneum, and acute cholecystitis due to stent occlusion are significant complications of EUS-GBD

\section{References}

1. Rana SS, Sharma R, Dhalaria L, Gupta R. Efficacy and safety of plastic versus lumen-apposing metal stents for transmural drainage of walled-off necrosis: a retrospective single-center study. Ann Gastroenterol 2020;33:426-432.

2. Sharma V, Rana SS, Bhasin DK. Endoscopic ultrasound guided interventional procedures. World J Gastrointest Endosc 2015;7:628-642.

3. Guo J, Saftoiu A, Vilmann P, et al. A multi-institutional consensus on how to perform endoscopic ultrasound-guided peri-pancreatic fluid collection drainage and endoscopic necrosectomy. Endosc Ultrasound 2017;6:285-291.

4. Bruno MJ. LAMS for all pancreatic fluid collections? Endosc Int Open 2020;8:E1161-E1162.

5. Boregowda U, Umapathy C, Nanjappa A, et al. Endoscopic ultrasound guided gallbladder drainage - is it ready for prime time? World J Gastrointest Pharmacol Ther 2018;9:47-54.

6. Posner H, Widmer J. EUS guided gallbladder drainage. Transl Gastroenterol Hepatol 2020;5:41.

7. Iino C, Shimoyama T, Igarashi T, et al. Comparable efficacy of endoscopic transpapillary gallbladder drainage and percutaneous transhepatic gallbladder drainage in acute cholecystitis. Endosc Int Open 2018;6:E594-E601.

8. Maruta A, Iwata K, Iwashita T, et al. Factors affecting technical success of endoscopic transpapillary gallbladder drainage for acute cholecystitis. J Hepatobiliary Pancreat Sci 2020;27:429-436.

9. Ogawa O, Yoshikumi H, Maruoka N, et al. Predicting the success of endoscopic transpapillary gallbladder drainage for patients with acute cholecystitis during pretreatment evaluation. Can J Gastroenterol 2008;22:681-685.

10. Luk SW, Irani S, Krishnamoorthi R, Wong Lau JY, Wai Ng EK, Teoh AY. Endoscopic ultrasound-guided gallbladder drainage versus percutaneous cholecystostomy for high risk surgical patients with acute cholecystitis: a systematic review and meta- 
analysis. Endoscopy 2019;51:722-732.

11. Teoh AYB, Kitano M, Itoi T, et al. Endosonography-guided gallbladder drainage versus percutaneous cholecystostomy in very high-risk surgical patients with acute cholecystitis: an international randomised multicentre controlled superiority trial (DRAC 1). Gut 2020;69:1085-1091.

12. Teoh AY, Perez-Miranda M, Kunda R, et al. Outcomes of an international multicenter registry on EUS-guided gallbladder drainage in patients at high risk for cholecystectomy. Endosc Int Open 2019;7:E964-E973.

13. Krishnamoorthi R, Jayaraj M, Thoguluva Chandrasekar V, et al. EUS-guided versus endoscopic transpapillary gallbladder drainage in high-risk surgical patients with acute cholecystitis: a systematic review and meta-analysis. Surg Endosc 2020;34:19041913.

14. Mori Y, Itoi T, Baron TH, et al. Tokyo Guidelines 2018: management strategies for gallbladder drainage in patients with acute cholecystitis (with videos). J Hepatobiliary Pancreat Sci 2018;25:87-95.

15. Jones MW, Hannoodee S, Young M. Anatomy, abdomen and pelvis, gallbladder. StatPearls Publishing: Treasure Island (FL); 2020.

16. Mahadevan V. Anatomy of the gallbladder and bile ducts. Surgery (Oxford) 2014.

17. Chan JHY, Teoh AYB. Current status of endoscopic gallbladder drainage. Clin Endosc 2018;51:150-155.

18. Itoi T, Itokawa F, Kurihara T. Endoscopic ultrasonographyguided gallbladder drainage: actual technical presentations and review of the literature (with videos). J Hepatobiliary Pancreat Sci 2011;18:282-286.

19. Widmer J, Singhal S, Gaidhane M, Kahaleh M. Endoscopic ultrasound-guided endoluminal drainage of the gallbladder. Dig Endosc 2014;26:525-531.

20. Crino SF, Rimbaș M, Gabbrielli A, Larghi A. Endoscopic ultrasound guided gallbladder interventions: a review of the current literature. J Gastrointestin Liver Dis 2019;28:339-347.

21. Amin S, Sethi A. EUS-guided gallbladder drainage. In: Adler DG (editor) Interventional endoscopic ultrasound. Springer International Publishing: Cham; 2019, pp. 35-43.

22. Sagami R, Hayasaka K, Ujihara T, et al. Feasibility of endoscopic ultrasound-guided gallbladder drainage for acute cholecystitis patients receiving antithrombotic therapy. Ann Gastroenterol 2020;33:391-397.

23. Anderloni A, Attili F, Sferrazza A, et al. EUS-guided gallbladder drainage using a lumen-apposing self-expandable metal stent in patients with coagulopathy or anticoagulation therapy: a case series. Endosc Int Open 2017;5:E1100-E1103.

24. Itoi T, Binmoeller KF, Shah J, et al. Clinical evaluation of a novel lumen-apposing metal stent for endosonography-guided pancreatic pseudocyst and gallbladder drainage (with videos). Gastrointest Endosc 2012;75:870-876.

25. Law R, Baron TH. Endoscopic ultrasound-guided gallbladder drainage. Gastrointest Endosc Clin N Am 2018;28:187-195.

26. James TW, Baron TH. EUS-guided gallbladder drainage: A review of current practices and procedures. Endosc Ultrasound 2019;8:S28-S34.

27. Adler DG. EUS-guided gallbladder drainage: Current status and future prospects. Endosc Ultrasound 2018;7:1-3.

28. Anderloni A, Buda A, Vieceli F, Khashab MA, Hassan C, Repici A. Endoscopic ultrasound-guided transmural stenting for gallbladder drainage in high-risk patients with acute cholecystitis: a systematic review and pooled analysis. Surg Endosc 2016;30:5200-5208.

29. Rana SS, Shah J, Kang M, Gupta R. Complications of endoscopic ultrasound-guided transmural drainage of pancreatic fluid collections and their management. Ann Gastroenterol 2019;32:441-450.

30. Sobani ZA, Ling C, Rustagi T. Endoscopic ultrasound-guided gallbladder drainage. Dig Dis Sci 2020 Aug 4 [Online ahead of print]. doi: 10.1007/s10620-020-06520-y

31. Manta R, Zulli C, Zullo A, et al. Endoscopic ultrasound-guided gallbladder drainage for acute cholecystitis with a silicone-covered nitinol short bilaterally flared stent: a case series. Endosc Int Open 2017;5:E1111-E1115.

32. Perez-Miranda M. Technical considerations in EUS-guided gallbladder drainage. Endosc Ultrasound 2018;7:79-82.

33. Kalva NR, Vanar V, Forcione D, Bechtold ML, Puli SR. Efficacy and safety of lumen apposing self-expandable metal stents for EUS guided cholecystostomy: a meta-analysis and systematic review. Can J Gastroenterol Hepatol 2018;2018:7070961.

34. Teoh AYB. Endoscopic ultrasound-guided anastomosis: Is it ready for prime time? J Gastroenterol Hepatol 2020;35:1288-1293.

35. Ogura T, Higuchi K. Endoscopic ultrasound-guided gallbladder drainage: current status and future prospects. Dig Endosc 2019;31 Suppl 1:55-64.

36. Teoh AYB. EUS-guided gallbladder drainage: is it so easy? Endosc Int Open 2020;8:E97-E98.

37. Xu MM, Kahaleh M. EUS-guided transmural gallbladder drainage: a new era has begun. Therap Adv Gastroenterol 2016;9:138-140.

38. Teoh AYB. Outcomes and limitations in EUS-guided gallbladder drainage. Endosc Ultrasound 2019;8:S40-S43.

39. Perisetti A, Deneke M, Thandassery R, Tharian B. Role of different types of stents in EUS-guided gallbladder drainage. Gastrointest Endosc 2020;91:961.

40. Yang MJ, Hwang JC, Yoo BM, KIM JH. Tips for dealing with common beginner's mistakes made during endoscopic ultrasoundguided gallbladder drainage. Dig Dis 2020;38:542-546.

41. Jain D, Bhandari BS, Agrawal N, Singhal S. Endoscopic ultrasoundguided gallbladder drainage using a lumen-apposing metal stent for acute cholecystitis: a systematic review. Clin Endosc 2018;51:450-462.

42. Imai $\mathrm{H}$, Kitano $\mathrm{M}$, Omoto $\mathrm{S}$, et al. EUS-guided gallbladder drainage for rescue treatment of malignant distal biliary obstruction after unsuccessful ERCP. Gastrointest Endosc 2016;84:147-151.

43. Saumoy M, Tyberg A, Brown E, et al. Successful cholecystectomy after endoscopic ultrasound gallbladder drainage compared with percutaneous cholecystostomy, can it be done? J Clin Gastroenterol 2019;53:231-235.

44. Kim JJ, Hiotis SP, Sur MD. Gastric reflux into the gallbladder after EUS-guided stenting-letter to the editor regarding "EUS-guided versus percutaneous gallbladder drainage: isn't it time to convert?". J Clin Gastroenterol 2019;53:392-393.

45. Kamata K, Takenaka M, Kitano M, et al. Endoscopic ultrasoundguided gallbladder drainage for acute cholecystitis: Long-term outcomes after removal of a self-expandable metal stent. World J Gastroenterol 2017;23:661-667.

46. Chan SM, Teoh AYB, Yip HC, Wong VWY, Chiu PWY, Ng EKW. Feasibility of per-oral cholecystoscopy and advanced gallbladder interventions after EUS-guided gallbladder stenting (with video). Gastrointest Endosc 2017;85:1225-1232.

47. Zou YP, Du JD, Li WM, et al. Gallstone recurrence after successful percutaneous cholecystolithotomy: a 10-year follow-up of 439 cases. Hepatobiliary Pancreat Dis Int 2007;6:199-203. 